Das Dossier Psychotherapie war neben mehreren anderen - z.B. Radiologie, wo wir weiter versuchen, eine Lösung zu finden, die den Erwartungen der Radiologen und der Nichtradiologen gerecht wird - eines der wichtigen und schwierigen Themen in den ersten Monaten dieses Jahres. Die Verhandlungen mit dem BAG, über die unten Bilanz gezogen wird, waren von einer sehr konstruktiven Zusammenarbeit zwischen den Instanzen der FMH und der betroffenen Fachgesellschaften geprägt. Wir haben Hans Kurt, den Präsidenten der Dachorganisation der Psychiater FMPP, um ein Fazit gebeten, und wir danken ihm herzlich für seinen interessanten Beitrag. Die von der Krankenpflegeleistungsverordnung (KLV) anerkannten Psychotherapien haben ihre Wirksamkeit zweifellos unter Beweis gestellt. Sie sind unverzichtbare therapeutische Instrumente der psychiatrischen Behandlung und müssen auch in Zukunft zur Verfügung stehen.

Der Text von Hans Kurt beweist, dass die vom BAG vorgeschlagenen Änderungen der KLV einige Fragen offenlassen, bei denen die Aufmerksamkeit der gesamten Ärzteschaft gefordert ist. Es ist bedauerlich, dass die Versicherten bei diesen Änderungen von der Diskussion über ihre Behandlung ausgeschlossen werden und eine unproduktive Polarisierung zwischen Leistungsträgern und Versicherern aufrechterhalten wird. Wäre es nicht sinnvoller gewesen, dem Versicherten die Möglichkeit eines direkten Rekurses an eine paritätische Kommission zu geben, wenn seine Versicherung eine Pflegeleistung nicht übernehmen will?

Dr. med. René Raggenbass, Mitglied des Zentralvorstandes der FMH

\title{
Mehr Effizienz! Aber: mehr Kontrolle in der Psychotherapie?
}

Sie erinnern sich? Vor einem Jahr wurde die Komplementärmedizin aus der Grundversicherung gestrichen, und der damalige Vizedirektor des BAG kündigte die Überprüfung der Psychotherapie und der Rehabilitation an. Anstatt eines Dialoges mit den psychiatrischen Fachgesellschaften und der FMH erklärte das BAG die Psychotherapie für zu kostspielig, Behandlungen von 10 bis 12 Sitzungen seien ausreichend, alles andere sei Wellness, und überhaupt gehörten Befindlichkeitsstörungen nicht in den Behandlungskatalog der obligatorischen Krankenpflegeversicherung OKP. Es waren gerade diese entwertenden Aussagen, geprägt von Misstrauen gegenüber Psychiatern und anderen Ärzten, die mehr Ärger und Empörung auslösten als die Vorschläge, die später gemacht wurden.

Die psychiatrischen Fachgesellschaften, unterstützt von der FMH, warteten bis Ende März 2006, um schliesslich $\mathrm{zu}$ einem Hearing eingeladen $\mathrm{zu}$ werden. In der Krankenpflegeleistungsverordnung KLV sollen Artikel 2 und 3 zur Psychotherapie geändert werden, obwohl sich diese über Jahre bewährt haben. Die Neuregelung der Psychotherapie in der KLV beinhaltet eine «frühe» Meldung (nach der 6. Sitzung und vor der 10. Sitzung) und eine erneute Berichterstattung nach weiteren 30 Psychotherapiesitzungen (also nach insgesamt 40 anstatt 60 Stunden wie bisher) Damit wird die Rolle der Vertrauensärzte im Vergleich zu früher deutlich gestärkt. Die Neuerungen sollen einer Evaluation unterzogen werden (2007-2010). Es wird weiterhin zwischen integrierter psychiatrisch-psychotherapeutischer Behandlung und Psychotherapie im engeren Sinne - nur hier gilt die KLV - unterschieden. Haben wir vorgängig von Misstrauen gesprochen, ist nun von vermehrter Kontrolle und bürokratischem Mehraufwand auszugehen.

Fragen bleiben offen: Angaben über psychische Erkrankungen sind höchst sensible, schützenswerte Daten, nicht nur solche von Patientinnen und Patienten, sondern auch diejenigen von deren Familien und Angehörigen. Mehr Berichte bedeuten aber mehr Informationen. Es ist zurzeit mehr als fragwürdig, ob die Kassenorgane in der Lage und gewillt sind, ihre Pflichten bezüglich Datenschutz ernst $\mathrm{zu}$ nehmen. Werden die Vertrauensärzte die vielen Meldungen und Berichte angemessen zu sichten und zu beurteilen wissen? Werden sie der Schwierigkeit komplexer psychotherapeutischer Fragestellungen gerecht werden? Aus gesetzlicher Sicht verunmöglicht das KVG ein aufschiebendes Rekursverfahren. So bleibt die Frage, was bei der Ablehnung einer Behandlung geschieht. Muss ein Patient Monate bis Jahre auf eine Weiterbehandlung warten, bis sein Anspruch versicherungsgerichtlich geklärt ist? Eine psychiatrisch-psychotherapeutische Zweitbeurteilung in strittigen Entscheiden ist deshalb unabdingbar.

Es ist anzunehmen, dass den Einschränkungen in der Psychotherapie weitere in anderen Bereichen der Medizin folgen werden. Es gilt, Abschied zu nehmen von der ärztlichen Freiheit und der psychotherapeutischen Kunst. Darüber kann Trauer und Enttäuschung aufkommen, haben wir doch zunehmend eine kontrollierbare, möglichst kostengünstige Dienstleistung zu erbringen. Wir helfen bei Neuerungen, die zwischen staatlichen Organen, den Versicherern und uns partnerschaftlich erarbeitet worden sind, mit. Aber wir verlangen Arbeitsbedingungen, die uns eine korrekte, qualitativ gute Behandlung unserer Patientinnen und Patienten erlauben. Und wir können Neuerungen nur dann zustimmen, wenn sie nicht zu Lasten der Patienten fallen, insbesondere psychisch kranker Menschen, die krankheitsbedingt ihre Rechte selbst kaum einfordern und über keine mächtige Lobby verfügen.

Dr. med. Hans Kurt, Präsident FMPP/SGPP 